\title{
Blood exosomal miRNA profiling reveals the complexity of hepatocellular carcinoma and identifies potential biomarkers as differential diagnosis
}

\section{Langqing Sheng}

Xiangya hospital

Jiarong Li

Xiangya hospital

Hao Qin

3D Medicines Inc.

Ling Liu

Xiangya hospital

Dadong Zhang

3D Medicines Inc.

Qi Zhang

Xiangya hospital

Mengli Huang

3D Medicines Inc.

Xiaoli Li

Xiangya hospital

Xiaoya Xu

3D Medicines Inc

Yangnian Wei

Xiangya hospital

\section{Zishuo Chen}

3D Medicines Inc.

Hui Luo

Xiangya hospital

Jiyang Zhang

3D Medicines Inc.

Chenghui Zhou

Xiangya hospital

Hao Chen

3D Medicines Inc. 


\section{Zeguo Chen}

Xiangya hospital

\section{Fugen Li}

3D Medicines Inc.

Nianfeng Li ( $\sim 1535909540 @ q q . c o m$ )

Xiangya hospital https://orcid.org/0000-0003-3442-2781

\section{Research article}

Keywords: Blood exosomal miRNAs, biomarker, differential diagnosis, alcohol consumption habit, hepatocellular carcinoma

Posted Date: January 29th, 2020

DOI: https://doi.org/10.21203/rs.2.22220/v1

License: (c) (i) This work is licensed under a Creative Commons Attribution 4.0 International License. Read Full License 


\section{Abstract}

Hepatocellular carcinoma $(\mathrm{HCC})$ is one of leading causes of cancer-related deaths worldwide, but there is a shortage of effective biomarkers for its diagnosis. We aimed to explore exosomal miRNAs as potential biomarkers for HCC diagnosis. After the eliminations, this study included 89 patients from Xiangya Hospital of Central South University between August 2017 and May 2018. The principal component analysis (PCA) analysis suggested that daily alcohol consumption could alter the blood exosomal miRNA profiles of HBV positive non-HCC patients through miR-3168 and miR-223-3p. The miRNA profiles also revealed the tumor stages of HCC patients. High expression of miR-455-5p and miR-30c-5p, which significantly correlated with better overall survival in tumor tissues, could also be detected in blood exosomes. Two pairs of miRNAs (miR-584-5p/miR-106-3p and miR-628-3p/miR-941) showed $94.1 \%$ sensitivity and $68.4 \%$ specificity to differentiate HCC patients from non-HCC patients. The specificity of the combination was substantially influenced by alcohol consumption habits. This study suggested that blood exosomal miRNAs can be used as new non-invasive diagnostic tools for HCC. However, their accuracy could be affected by tumor stage and alcohol consumption habits.

\section{Introduction}

Hepatocellular carcinoma (HCC) is one of the leading causes of cancer-associated mortality worldwide, especially in East and South Asia [1, 2]. In China, more than 460,000 new cases of HCC were diagnosed with 422,100 deaths in 2015 [3]. Due to its insidious onset without specific early symptoms, most of the HCC patients are diagnosed with advanced stage cancer. The lack of effective early diagnosis methods for HCC is a major challenge to improving the outcomes of these patients, who therefore lose the opportunity for therapeutic treatments, such as resection and sorafenib $[4,5]$. Hence, there is an urgent demand for the identification of potential biomarkers for early detection to cut down the high mortality of HCC.

It was reported that some tumor biomarkers in serum had been discovered and might play an important role in HCC diagnosis [6, 7]. At present, the serum a-fetoprotein (AFP) test is a common and important early diagnostic test for liver cancer, but this biomarker has a low specificity [8]. In addition, carbohydrate antigen (CA) 19-9 (CA19-9) and carcinoembryonic antigen (CEA) have been widely used as serum tumor markers for the clinical diagnosis of HCC [9]. However, the efficacy of these tumor markers in the clinical diagnosis of HCC remains unsatisfactory. In addition, candidate tumor biomarkers, such as circulating tumor cells (CTCs), serum cell-free DNA, non-coding RNAs, and microRNA, show limited progress in advanced $\mathrm{HCC}$ to date [4, 10-12]. In the face of the difficult problem of exploring biomarkers for diagnosis of HCC, it is particularly important to adopt new techniques and study related interference factors.

Exosomes, which are 50- to 150-nm membrane microvesicles secreted by a number of cell types, have been found in many types of body fluids, including saliva, plasma, breast milk, urine, and malignant effusions [13]. It has been reported that exosomes serve as the key regulator of the tumor microenvironment by promoting HCC occurrence and progress [14]. The transfer of exosomes from 
primary tumors to the circulatory system has been demonstrated in various models [15], and many studies have indicated that cancer-associated exosomal miRNAs participate in the regulation of cancer cell growth, metastasis, and drug resistance $[16,17]$. This rapidly expanding field includes studies investigating opportunities for developing serum-derived exosomal contents as new biomarkers in cancer diagnosis, treatment, and drug resistance.

Considering the frequency of late diagnosis in $\mathrm{HCC}$, biomarkers as potential prognostic indicators and their complexity are worthy of further investigation. Several studies have reported application of exosomal miRNAs as biomarkers in early detection, diagnosis, and therapy in $\operatorname{HCC}[4,18,19]$. However, the previous research was on a small scale and showed differences in candidate biomarkers using realtime PCR or microarrays, which are classic methods for miRNA expression analysis and can detect only known and limited miRNAs. In previous studies, there was a lack of comprehensive microRNA (miRNA) expression profiling. Such comprehensive profiling has proven useful in diagnosing and monitoring the development and progression of tumors. Recently, with the rapid development of next-generation sequencing, microRNA sequencing (miRNA-seq) has begun to offer increased specificity and sensitivity in miRNA profiling [20]. In particular, it possesses the ability to identify novel miRNAs, which has significantly enabled rapid profiling and deep investigation of miRNAs. Therefore, we adopted two relatively large population cohorts (including HBV-infected individuals, liver cirrhosis patients, and HCC patients) for exosomal miRNA profiling using microRNA sequencing. Cohort analysis of blood exosomal miRNA profiling was performed and revealed the complexity caused by drinking alcohol and potential biomarkers of differential diagnosis in hepatocellular carcinoma. This work was the first study revealing how exosomal miRNA biomarkers are made more complex by drinking alcohol in the differential diagnosis of HCC using exosomal miRNA sequencing.

\section{Materials And Methods}

\subsection{Patient clinical information and clinical sample collection}

This study was approved by the Ethics Committee of Xiangya Hospital of Central South University (No. 201803818). There were 136 patients enrolled for this study between August 2017 and May 2018. After the elimination of pathology samples from patients with non-HCC malignant tumors and hemolysis, this study included 89 patients, comprising 51 patients with hepatocellular carcinoma (HCC) confirmed by surgical pathology, 20 liver cirrhosis patients, and 18 HBV-infected individuals. These patients were divided into two cohorts (cohort 1 and 2). Detailed clinical characterization of these patients is shown in Table 1. We collected data about the patients' age, sex, cirrhosis, tumor stage, alcohol consumption, and levels of various tumor biomarkers (AFP, CA19-1, and CEA) (Table 1). All of the individuals at the Xiangya Hospital of Central South University gave their written consent for their plasma samples and pathology information to be used in this research.

The blood samples of these patients were collected in vacutainers with anticoagulant (REF367863, BD, USA) and then shipped at $4^{\circ} \mathrm{C}$ after collection. When we received the blood samples we first centrifuged 
them at $4^{\circ} \mathrm{C}(1600 \times \mathrm{g}, 10$ minutes $)$. After this, we determined and recorded the hemolysis level. Hemolysis levels beyond 4 plasma samples were not used in this study. Followed by a second centrifugation at $4^{\circ} \mathrm{C}$ $\left(16000 \times \mathrm{g}, 10\right.$ minutes), we finally transferred the supernatants to new tubes and stored them at $-80^{\circ} \mathrm{C}$ for further study.

\subsection{Plasma exosome isolation}

After samples were incubated and centrifuged at $4^{\circ} \mathrm{C}(12,000 \times \mathrm{g}, 10$ minutes), we transferred the supernatants into a $0.45-\mu \mathrm{m}$ tube filter (Costar, CLS8163-100EA, Corning, USA), centrifuged them at $4^{\circ} \mathrm{C}$ (12,000×g, 5 minutes), and then filtered them with 0.22- $\mu \mathrm{m}$ tube filters (Costar, CLS8161-100EA, USA). The supernatants were transferred into fresh $1.5-\mathrm{mL}$ tubes. Plasma exosome isolation was performed as previously described [21]. We then added one-quarter volume exosome isolation reagent (L3525, 3DMed, Shanghai, China). We gently vortexed the mixtures and incubated them for 30 minutes at $4^{\circ} \mathrm{C}$, and then centrifuged them at $4^{\circ} \mathrm{C}(4700 \times \mathrm{g}, 30$ minutes). The supernatants were discarded, and the precipitates, containing the exosomes, were resuspended in $200 \mu \mathrm{L}$ phosphate-buffered saline (PBS).

\subsection{Characterization of exosomes-scanning electron microscopic analyses}

Scanning electron microscopy was performed as previously described [22]. Briefly, exosomes were suspended in PBS before fixing in 5\% glutaraldehyde. Then, exosomes were immobilized in 1\% 0sO4 and were dehydrated with various concentrations of ethanol. Subsequently, samples underwent drying at room temperature for $24 \mathrm{~h}$ and were finally analyzed by SEM (SU8020, Hitachi High-Technologies, Japan).

\subsection{Quantification of exosomes protein}

Exosome protein extraction was done with exosome isolation reagent (N3525, 3DMed, Shanghai, China). Then, exosomes were lysed in $200 \mu \mathrm{L}$ of lysis buffer (P0013B, Beyotime, Shanghai, China). Equal amount of proteins were run on a $4 \%-20 \%$ SDS-PAGE gel (\#4561095, Bio-Rad, USA). Proteins were transferred onto a PVDF membrane (Millipore), then incubated at room temperature for 2 hours with primary antibodies anti-CD9 (1:500 dilution, ab92726, Abcam, England) and anti-CD63 (1:2000 dilution, ab68418, Abcam, England). Anti-rabbit IgGs (sc-2004, Santa Cruz, USA) were added and blots were incubated at room temperature for 40 minutes. Blots were visualized using an enhanced chemiluminescence system according to the manufacturer's protocol (Tanon-5200Multi, Shanghai, China). 
Exosome miRNAs were extracted by the miRNeasy Serum/Plasma Kit according to the manufacturer's instructions. (217184, QIAGEN, Shanghai, China). The yield, distribution, and quality of miRNAs were analyzed by the Agilent 2100 bioanalyzer using Small RNA Chips (5067-1548, Agilent, USA).

\subsection{Small RNA sequencing libraries preparation}

A total of $100 \mathrm{ng}$ RNA per sample was used to construct the miRNA library using the NEBNext Multiplex Small RNA Library Prep Set for Illumina (E7300L, NEB, USA) according to the manufacturer's protocol. Eighteen cycles of PCR were performed after first strand cDNA synthesis. The DNA libraries were purified by NucleoSpin Gel and PCR Clean-up (740609.50, QIAGEN, Shanghai, China). The quality and distribution of libraries were analyzed using the LabChip ${ }^{\circledR}$ GX Touch $^{\mathrm{TM}} 24$ Nucleic Acid Analyzer. The pooled libraries were sequenced using an Illumina HiSeq PE150 analyzer.

\subsection{Bioinformatics analysis of miRNA-seq data}

The miRNA-seq data were quantified and tested for differential expression with DESeq2 package in $\mathrm{R} /$ Bioconductor [23]. After raw data cleaning, sequences with a length less than 30nt were mapped to hg19 by BWA [24] 0.7.12-r1039. The raw expression of a miRNA was defined by the number of reads mapped to the loci of the mature miRNA, which was obtained from miRBase [25] v21. Due to the limited sequencing depth, only those miRNAs which had at least one read in each sample were considered in the following analysis. Before comparing the expression between samples, the raw expression was normalized. The 75th percentile expression of samples was calculated as the size factor. The miRNA profiling was normalized using counts per million (CPM) mappable miRNA sequences. The method is described in DESeq2 [23].

\subsection{Statistical analysis}

To scale for miRNA expression, principal component analysis (PCA) was performed, wherein the normalized expression levels of each miRNA were scaled by dividing the expression levels by the maximum expression levels of the miRNAs. PCA was then performed on the matrix constructed by the scaled expression levels, using the prcomp function in R 3.3.3.

To test the performance of biomarkers, the area under the curve (AUC) was calaulated using R/Bioconductor pROC (v1.12.1) package. Clinicopathologic diagnoses were used as the gold standard to assess the diagnostic accuracy of a group of exosomal miRNAs by AUC. Kaplan-Meier plot analysis of The Cancer Genome Atlas (TCGA) data was performed using the online bioinformatics tool, OncoLnc (http://www.oncolnc.org/). $\mathrm{P}<0.05$ was considered statistically significant. 


\section{Results}

\subsection{HBV-positive hepatocarcinoma (HCC) patients did not show differential blood exosomal miRNA profiles compared with HBV-positive non-HCC patients}

To study the blood exosomal miRNA profiles, $89 \mathrm{HBV}$ positive patients were enrolled and their blood was collected between August 2017 and May 2018. Among these patients, there were 51 patients diagnosed with hepatocellular carcinoma (HCC), 20 liver cirrhosis patients, and $18 \mathrm{HBV}$-infected individuals. All of the patients were distributed to two cohorts (cohort 1 and 2). The clinical information and statistics of the two cohorts are listed in Table 1.

To characterize the exosomes of plasma obtained from these patients, Western blotting (WB) and scanning electron microscopic (SEM) analysis were performed. SEM images of two representative samples are shown (Supplementary Figure S1a). Two characteristic proteins of exosomes are CD9 and CD63. The protein levels in four representative samples are identified (Supplementary Figure S1b). These results suggest that exosomes of the plasma from these patients were successfully obtained by this method.

First, all of the patients were pooled to explore the potential differential features between cancer patients and non-cancer patients. Principal component analysis (PCA) was performed to identify the major variations, which might reveal their biological nature (Fig. 1). The distribution of cancer patients and noncancer patients generally overlapped in the top five principal components (PC) and their combinations. The PCA result suggested that multiple other factors could more significantly affect the blood exosomal miRNA profiles than can the biological difference between HBV infected HCC and non-HCC patients.

\subsection{Daily alcohol consumption could alter the blood exosomal miRNA profiles of HBV positive non-HCC patients}

On the coordinates of PC 1 and PC 2, non-HCC patients who had a daily alcohol consumption habit tended to distribute in the bottom right corner (Fig. 2A), suggesting that alcohol consumption might shift the blood exosomal miRNA profile. To identify the key miRNAs, which are expressed in the response to alcohol consumption, a two-dimensional coordinate was constructed by the weights of each miRNA in PC1 and PC2 (Fig. 2B). In this coordinate, miR-3168 and miR-223-3p were spotted as outliers in the bottom right corner. Both miRNAs showed significantly higher levels in non-HCC patients with daily alcohol consumption (Fig. 2C and 2D). However, non-HCC patients who had a daily alcohol consumption habit and HCC patients (drinking or non-drinking) showed comparable blood exosomal miRNA levels.

\subsection{Blood exosomal miRNA profiles revealed the staging of tumor tissues}


On the coordinates constructed from PC1 and PC 2, the HCC patients were colored according to the Barcelona Clinic Liver Cancer (BCLC) tumor staging system [26] (Fig. 3A). Interestingly, patients with stage $\mathrm{C}$ HCC tumors were mainly distributed in the negative direction on the PC 1 coordinate, indicating that the vital miRNAs that contributed to the variation of PC 1 might reveal tumor staging (Fig. 3B). Since blood exosomes could derive from various types of cells, the clinical and miRNA-seq data of $372 \mathrm{HCC}$ tissue samples from TCGA were analyzed to discover the connection between tumor tissues and blood exosomes in further detail. A Cox regression model was performed on miRNAs which were expressed in all of the tissue samples. Then, the Cox regression results from the tumor tissues were integrated with the PC weights of the blood exosomal miRNAs (Fig. 3C). We found that 29 miRNAs possessed a positive weight of PC 1 larger than 0.1 (in the grey rectangle), while none of the miRNAs possessed a negative weight less than -0.05 , suggesting that the 29 miRNAs mainly contributed to the variation of PC 1 . Among the 29 miRNAs, 11 miRNAs showed significant or marginal p-values in the Cox regression model, and all of those miRNAs possessed negative hazard ratios. MiR-455-5p and miR-30c-5p were ranked among the top 5 miRNAs with positive weights. Both miRNAs were suggested to be tumor suppressors in various types of cancers [27,28], and showed significantly better overall survival in the patients with higher expression levels (Fig. 3D and 3E). Blood exosomal miRNAs are related to the stage of the tumor tissues, which might predict the survival of HCC patients. However, miRNAs from other tissues or cells might also influence the signals from tumor tissues.

\subsection{The accuracy of biomarkers to classify HCC and non-HCC patients from blood exosome miRNAs was affected by tumor stage and alcohol consumption habits}

Since blood exosomal miRNAs might derive from various sources, normalization of all miRNAs with the same size factor (or the same housekeeping miRNA) might not reveal the real difference of each miRNA between HCC and non-HCC patients. Similar to the normalization concept of GPCR, the quotient between a pair of miRNAs, where one is considered the target gene and the other one is considered the reference gene, was calculated. This exhaustive method was used to find the best reference gene to normalize each target gene, and their quotients were considered the candidate biomarkers. To avoid potential batch effects, the patients were allocated to two cohorts according to their experimental date. The accuracy of each pair of miRNAs were measured by area under the curve (AUC) of receiver operating characteristic (ROC). The pairs of miRNAs were ranked according to the average AUC between two cohorts, where the average AUC should be larger than 0.7 (Fig. 4A). The top two pairs of miRNAs were miR-628-3p/miR-941 and miR-584-5/miR-106b-3p, and their average AUCs were 0.7423 and 0.7421 , respectively. To distinguish HCC patients from non-HCC patients, miR-584-5p/miR-106b-3p showed $94.7 \%$ specificity under a cutoff of 1.5 , but the sensitivity was relatively low (37.2\%). Under a cutoff of 0.65 , miR-628-3p/miR-941 showed $76.5 \%$ sensitivity and $73.7 \%$ specificity. When we considered a sample which had either of the quotients higher than their cutoffs as HCC patients, the combination of two quotients raised the sensitivity to $94.1 \%$, while the specificity was maintained at $68.4 \%$ (Fig. 4B). Combining the two quotients increased 
the overall accuracy to $83.1 \%$, which was higher than that for miR-584-5p/miR-106b-3p (61.8\%) or miR$628-3 p / m i R-941$ (75.3\%).

While miR-584-5p/miR-106b-3p did not show any bias toward distinguishing HCC patients from non-HCC patients at any stages, among the HCC patients which could not be identified by miR-584-5p/miR-106b$3 p$, the HCC patients in stage A and B showed lower overall levels of miR-628-3p/miR-941 than did those in stage $C$, and thus they were more difficult distinguish from non-HCC patients (Fig. 4B and Supplementary Figure S2). Additionally, non-HCC patients with different alcohol consumption habits also showed effects on their levels of miR-628-3p/miR-941 (Fig. 4C). Non-HCC patients with daily alcohol consumption presented significantly higher levels of miR-628-3p/miR-941 than did those without daily alcohol consumption and were more likely to be identified as false-positive HCC patients (Supplementary Figure S3).

\section{Discussion}

It has been reported that miRNAs in blood could distinguish HBV-infected HCC patients from HBVinfected non-HCC patients [29], which suggests that the signals released from HCC can be observed. Mohamed et al. found that circulating miR-23a showed an accuracy level of $79.3 \%$ in diagnosing HCC patients with a sensitivity of $89.47 \%$ and a specificity of about $64.91 \%$ [30]. Zhu et al. adopted a diagnostic 2-miRNA panel to differentiate HCC from healthy controls (AUC $=0.823, P<0.0001$ ) and cirrhosis patients (AUC $=0.859, P<0.0001$ ) [31]. Huang et al. established a score comprising 5 blood miRNAs and a binary etiology variable that was capable of differentiating between cirrhotic patients and HCC patients (AUC $=72.5 \%, P<0.001$ ) [32]. These results bring some hope for the differential diagnosis of HCC, but they are still not satisfactory for the clinical demand. However, blood miRNAs can be derived from various sources. Exosomes are small extracellular vesicles released from living cells for intercellular communications, and they also reveal the metabolic events occurring inside the cells. Hence, studying the miRNA profiles from HCC exosomes could provide insights for research and clinical diagnosis.

The exosomal miRNA profiles showed that in HBV-positive patients, the signals from tumors or triggered by tumors might not be major factors able to shift the whole profiles to be significantly distinct from those of non-HCC patients. However, other clinical features could be revealed through exosomal miRNA profiling. Among these clinical features, alcohol consumption is known to cause several liver diseases, including alcoholic liver disease (ALD), alcoholic fatty liver, alcoholic hepatitis, and alcoholic liver cirrhosis [33]. Liver is the main organ responsible for metabolizing ethanol, and thus it has been considered for a long time a major organ damaged by the harmful use of alcohol [34]. The expression of a wide variety of miRNAs is potentially regulated by many factors, such as alcohol, but also diet, cigarette smoking, and other drugs [35]. To date, there has only been one previous study focusing on the role of alcohol-regulated miRNAs in HCC pathogenesis and progression. That study reported that alcohol consumption in patients with positive HBV and those with HCC regulates microRNAs (miR-944 and miR223-3p) from tumor tissue. These microRNAs likely play previously uncharacterized roles in the alcoholassociated carcinogenesis of HCC [36]. Our study showed that miR-3168 and miR-223-3p had 
significantly higher levels in non-HCC patients with daily alcohol consumption than those without daily alcohol consumption, suggesting that daily alcohol consumption could alter the blood exosomal miRNA profiles of HBV positive non-HCC patients. MiR-223-3p, which from tumor tissue was reported to play a role in the alcohol-associated carcinogenesis of HCC, was discovered as a blood exosomal biomarker of alcohol consumption in our study. In addition, it has also been reported that when treated by alcohol, the miR-223-3p level in tumor cell lines and in the immortalized liver cell line L02 increased [36]. A similar trend was also revealed in blood exosomal miRNA. These results led to the idea that the function of miR223-3p in in the alcohol-associated carcinogenesis of HCC deserved further study.

The BCLC staging system classifies HCC patients into four stages that are highly correlated with prognosis [26]. Patients in early stage A or B are usually asymptomatic without vascular invasion or extrahepatic spread, while patients in advanced stage $C$ have either symptomatic tumors, vascular invasion, or extrahepatic spread, which causes poorer survival than early stages. Intriguingly, tumor stages are major contributors in shifting the miRNA profiles among tumor patients, especially between stage $C$ and early stages. We found that 29 miRNAs confer the major differences between stage $C$ and the early stages in blood exosomes, and $37.9 \%$ of them predict prognosis in the same direction in HCC tissues, suggesting that these miRNAs were mainly released from tumors. It is possible that miRNAs miR455-5p and miR-30c-5p could function as tumor suppressors [27, 28], with decreasing levels in stage $C$ tumors compared with the early stages. Hence, profiles of blood exosomal miRNAs could be harnessed to monitor the development of tumors.

Affected by multiple factors, accurately differentiating HBV-positive HCC patients and non-HCC patients by blood miRNA levels is difficult. Zhou et al. constructed a 7-miRNA model to distinguish HCC patients from non-HCC patients with chronic hepatitis $B$. The sensitivity was $79.1 \%$ and the specificity was $76.4 \%$ in a clinical trial of 934 patients [29]. However, this method is not significantly more accurate than current protein biomarkers, such as AFP, HSP, and GPC3 [37]. In this study, because the exosomes could be derived from a vast range of cells, conventional normalization methods for each miRNA might not be appropriate. Instead, the biomarkers were searched in pairs to find the best reference sequence for each miRNA. More biomarkers included in a model could increase the accuracy, but also could increase the risk of over-fitting. Due to the limitation of sample size, only the top two pairs of miRNAs were included in the model, and their performance was comparable to that of other miRNA models or protein-based biomarkers. The sensitivity of this model was $94.1 \%$, and advanced HCC patients were more easily detected than those in early stages. However, the specificity of this model was relatively low compared with other models or methods. One of the critical factors causing false positive results is alcohol consumption. Hence, to further improve the diagnostic accuracy, a more complex model constituted of biomarkers from multiple categories is necessary to eliminate the influence of various factors. Such a complex model will need to be validated in a large cohort of patients.

In conclusion, this study performed a cohort analysis of blood exosomal miRNA profiling to discover the complexity of differential diagnostic biomarkers for HCC and to confirm that exosomal miRNAs could be used as new biomarkers for HCC and liver cirrhosis. In addition, we showed that the accuracy of 
biomarkers to classify HCC and non-HCC patients from blood exosomal miRNAs could be affected by tumor stage and alcohol consumption habits. This study laid a foundation for understanding the complexity of blood exosomal microRNA profiles and for promoting the clinical application of blood exosomal microRNAs as biomarkers in the differential diagnosis of hepatocellular carcinoma.

\section{Abbreviations}

HCC: hepatocellular carcinoma; AUC: area under the curve; ROC: receiver operating characteristic; PCA: principal component analysis; PC: principal component; AFP: a-fetoprotein; CA19-9: carbohydrate antigen (CA) 19-9; CEA: carcinoembryonic antigen; WB: western blotting; SEM: scanning electron microscopic; miRNA: microRNA; ALD: alcoholic liver disease; BCLC: Barcelona Clinic Liver Cancer and TCGA: The Cancer Genome Atlas.

\section{Declarations}

\section{Ethics approval and consent to participate}

The study was approved by the ethics committee of Xiangya Hospital and informed consent of all patients or their relatives were signed.

\section{Consent for publication}

All the authors listed have approved the manuscript that is enclosed.

\section{Acknowledgements}

All the authors thank the authors would like to thank all of the patients and their families for their support of this study.

\section{Disclosure of Potential Conflicts of Interest}

All authors with 3D medicines affiliation are current or former employees. No potential conflicts of interest are disclosed by the other authors.

\section{Authors' Contributions}

N. Li, L. Sheng, J. Li, H. Qin, and D. Zhang designed the experiments. Li, L. Sheng, J. Li, H. Qin, D. Zhang, Q. Zhang, M. Huang, X. Xu, X. Li, Z. Chen, Y. Wei, H. Luo, C. Zhou, Z. Chen and F. Li performed the experiments. N. Li, H. Qin, D. Zhang, X. Xu, and Z. Chen carried out data analysis. Y. Zhang, N. Li, L. Sheng, J. Li, H. Qin, and D. Zhang wrote the manuscript.

\section{Funding}

The study is not supported by any funding. 
Data Availability Statement

The data used to support the findings of this study are available from the corresponding author upon request.

\section{References}

1. Hashim D, Boffetta P, La Vecchia C, Rota M, Bertuccio P, Malvezzi M, Negri E: The global decrease in cancer mortality: trends and disparities. Ann Oncol 2016, 27(5):926-933.

2. Bertuccio P, Turati F, Carioli G, Rodriguez T, La Vecchia C, Malvezzi M, Negri E: Global trends and predictions in hepatocellular carcinoma mortality. J Hepatol 2017, 67(2):302-309.

3. Chen W: Cancer statistics: updated cancer burden in China. Chin J Cancer Res 2015, 27(1):1.

4. Xue X, Zhao Y, Wang X, Qin L, Hu R: Development and validation of serum exosomal microRNAs as diagnostic and prognostic biomarkers for hepatocellular carcinoma. J Cell Biochem 2018.

5. Peck-Radosavljevic M: Drug therapy for advanced-stage liver cancer. Liver Cancer 2014, 3(2):125131.

6. Tsuchiya N, Sawada Y, Endo I, Saito K, Uemura Y, Nakatsura T: Biomarkers for the early diagnosis of hepatocellular carcinoma. World J Gastroenterol 2015, 21(37):10573-10583.

7. Song $P$, Tang $Q$, Feng $X$, Tang W: Biomarkers: evaluation of clinical utility in surveillance and early diagnosis for hepatocellular carcinoma. Scand J Clin Lab Invest Supp/ 2016, 245:S70-76.

8. Jeon Y, Jang ES, Choi YS, Kim JW, Jeong SH: Glypican-3 level assessed by the enzyme-linked immunosorbent assay is inferior to alpha-fetoprotein level for hepatocellular carcinoma diagnosis. Clin Mol Hepatol 2016, 22(3):359-365.

9. Banales JM, Inarrairaegui M, Arbelaiz A, Milkiewicz P, Muntane J, Munoz-Bellvis L, La Casta A, Gonzalez LM, Arretxe E, Alonso $C$ et al: Serum metabolites as diagnostic biomarkers for cholangiocarcinoma, hepatocellular carcinoma and primary sclerosing cholangitis. Hepatology 2018.

10. Li J, Han X, Yu X, Xu Z, Yang G, Liu B, Xiu P: Clinical applications of liquid biopsy as prognostic and predictive biomarkers in hepatocellular carcinoma: circulating tumor cells and circulating tumor DNA. J Exp Clin Cancer Res 2018, 37(1):213.

11. Lee YR, Kim G, Tak WY, Jang SY, Kweon YO, Park JG, Lee HW, Han YS, Chun JM, Park SY et al: Circulating exosomal non-coding RNAs as prognostic biomarkers in human hepatocellular carcinoma. Int J Cancer 2018.

12. Wang Y, Zhang C, Zhang P, Guo G, Jiang T, Zhao X, Jiang J, Huang X, Tong H, Tian Y: Serum exosomal microRNAs combined with alpha-fetoprotein as diagnostic markers of hepatocellular carcinoma. Cancer Med 2018, 7(5):1670-1679.

13. Lasser C, Alikhani VS, Ekstrom K, Eldh M, Paredes PT, Bossios A, Sjostrand M, Gabrielsson S, Lotvall $\mathrm{J}$, Valadi H: Human saliva, plasma and breast milk exosomes contain RNA: uptake by macrophages. 
Journal of translational medicine 2011, 9:9.

14. Sun F, Wang JZ, Luo JJ, Wang YQ, Pan Q: Exosomes in the Oncobiology, Diagnosis, and Therapy of Hepatic Carcinoma: A New Player of an Old Game. Biomed Res Int 2018, 2018:2747461.

15. Suetsugu A, Honma K, Saji S, Moriwaki H, Ochiya T, Hoffman RM: Imaging exosome transfer from breast cancer cells to stroma at metastatic sites in orthotopic nude-mouse models. Advanced drug delivery reviews 2013, 65(3):383-390.

16. Peinado H, Aleckovic M, Lavotshkin S, Matei I, Costa-Silva B, Moreno-Bueno G, Hergueta-Redondo M, Williams C, Garcia-Santos G, Ghajar C et al: Melanoma exosomes educate bone marrow progenitor cells toward a pro-metastatic phenotype through MET. Nature medicine 2012, 18(6):883-891.

17. Yu LX, Zhang BL, Yang Y, Wang MC, Lei GL, Gao Y, Liu H, Xiao CH, Xu JJ, Qin H et al: Exosomal microRNAs as potential biomarkers for cancer cell migration and prognosis in hepatocellular carcinoma patient-derived cell models. Oncology reports 2019, 41(1):257-269.

18. Suehiro T, Miyaaki H, Kanda Y, Shibata H, Honda T, Ozawa E, Miuma S, Taura N, Nakao K: Serum exosomal microRNA-122 and microRNA-21 as predictive biomarkers in transarterial chemoembolization-treated hepatocellular carcinoma patients. Oncol Lett 2018, 16(3):3267-3273.

19. Pan JH, Zhou H, Zhao XX, Ding H, Li W, Qin L, Pan YL: Role of exosomes and exosomal microRNAs in hepatocellular carcinoma: Potential in diagnosis and antitumour treatments (Review). Int $\mathrm{J} \mathrm{Mol}$ Med 2018, 41(4):1809-1816.

20. Buitrago DH, Patnaik SK, Kadota K, Kannisto E, Jones DR, Adusumilli PS: Small RNA sequencing for profiling microRNAs in long-term preserved formalin-fixed and paraffin-embedded non-small cell lung cancer tumor specimens. PloS one 2015, 10(3):e0121521.

21. Zhang JT, Qin H, Man Cheung FK, Su J, Zhang DD, Liu SY, Li XF, Qin J, Lin JT, Jiang BY et al: Plasma extracellular vesicle microRNAs for pulmonary ground-glass nodules. Journal of extracellular vesicles 2019, 8(1):1663666.

22. Manri C, Yokoi T, Nishida H: Size-Selective Harvesting of Extracellular Vesicles for Strategic Analyses Towards Tumor Diagnoses. Appl Biochem Biotechnol 2017, 182(2):609-623.

23. Love MI, Huber W, Anders S: Moderated estimation of fold change and dispersion for RNA-seq data with DESeq2. Genome Bio/ 2014, 15(12):550.

24. Li H, Durbin R: Fast and accurate short read alignment with Burrows-Wheeler transform. Bioinformatics 2009, 25(14):1754-1760.

25. Griffiths-Jones S, Saini HK, van Dongen S, Enright AJ: miRBase: tools for microRNA genomics. Nucleic Acids Res 2008, 36(Database issue):D154-158.

26. Llovet JM, Bru C, Bruix J: Prognosis of hepatocellular carcinoma: the BCLC staging classification. Semin Liver Dis 1999, 19(3):329-338.

27. Lai YH, Chen J, Wang XP, Wu YQ, Peng HT, Lin XH, Wang WJ: Collagen triple helix repeat containing-1 negatively regulated by microRNA-30c promotes cell proliferation and metastasis and indicates poor prognosis in breast cancer. J Exp Clin Cancer Res 2017, 36(1):92. 
28. Sandoval-Borquez A, Polakovicova I, Carrasco-Veliz N, Lobos-Gonzalez L, Riquelme I, Carrasco-Avino G, Bizama C, Norero E, Owen GI, Roa JC et al: MicroRNA-335-5p is a potential suppressor of metastasis and invasion in gastric cancer. Clin Epigenetics 2017, 9:114.

29. Zhou J, Yu L, Gao X, Hu J, Wang J, Dai Z, Wang JF, Zhang Z, Lu S, Huang X et al: Plasma microRNA panel to diagnose hepatitis B virus-related hepatocellular carcinoma. J Clin Oncol 2011, 29(36):47814788.

30. Mohamed AA, Ali-Eldin ZA, Elbedewy TA, El-Serafy M, Ali-Eldin FA, AbdelAziz H: MicroRNAs and clinical implications in hepatocellular carcinoma. World J Hepatol 2017, 9(23):1001-1007.

31. Zhu HT, Liu RB, Liang YY, Hasan AME, Wang HY, Shao Q, Zhang ZC, Wang J, He CY, Wang F et al: Serum microRNA profiles as diagnostic biomarkers for HBV-positive hepatocellular carcinoma. Liver Int 2017, 37(6):888-896.

32. Huang YH, Liang KH, Chien RN, Hu TH, Lin KH, Hsu CW, Lin CL, Pan TL, Ke PY, Yeh CT: A Circulating MicroRNA Signature Capable of Assessing the Risk of Hepatocellular Carcinoma in Cirrhotic Patients. Sci Rep 2017, 7(1):523.

33. Santos S, Mattos AA, Guimaraes MM, Boger BS, Coral GP: Alcohol Consumption Influences Clinical Outcome in Patients Admitted to a Referral Center for Liver Disease. Ann Hepatol 2018, 17(3):470475.

34. Rocco A, Compare D, Angrisani D, Sanduzzi Zamparelli M, Nardone G: Alcoholic disease: liver and beyond. World J Gastroenterol 2014, 20(40):14652-14659.

35. Xu T, Li L, Hu HQ, Meng XM, Huang C, Zhang L, Qin J, Li J: MicroRNAs in alcoholic liver disease: Recent advances and future applications. J Cell Physiol 2018, 234(1):382-394.

36. Zheng H, Zou AE, Saad MA, Wang XQ, Kwok JG, Korrapati A, Li P, Kisseleva T, Wang-Rodriguez J, Ongkeko WM: Alcohol-dysregulated microRNAs in hepatitis B virus-related hepatocellular carcinoma. PLoS One 2017, 12(5):e0178547.

37. Zhao YJ, Ju Q, Li GC: Tumor markers for hepatocellular carcinoma. Mol Clin Oncol 2013, 1(4):593598.

\section{Figures}




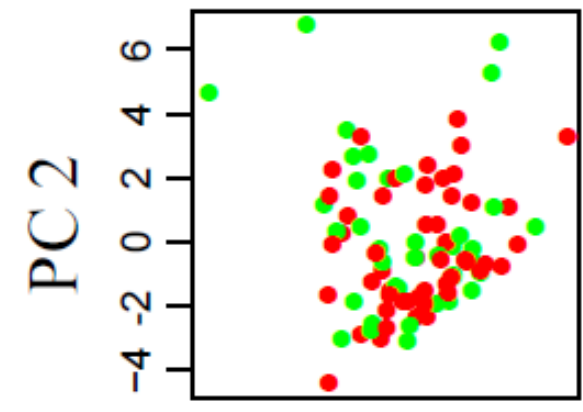

\section{Cancer patients \\ Non-cancer patients}


Figure 1

Blood exosomal miRNA profiles did not show distinctions between hepatocellular carcinoma (HCC) patients and non-HCC patients The top 5 PCs of blood exosomal miRNA profiles from HCC patients and non-HCC patients is shown. The red represents HCC patients, and green represents non-HCC patients. 
A

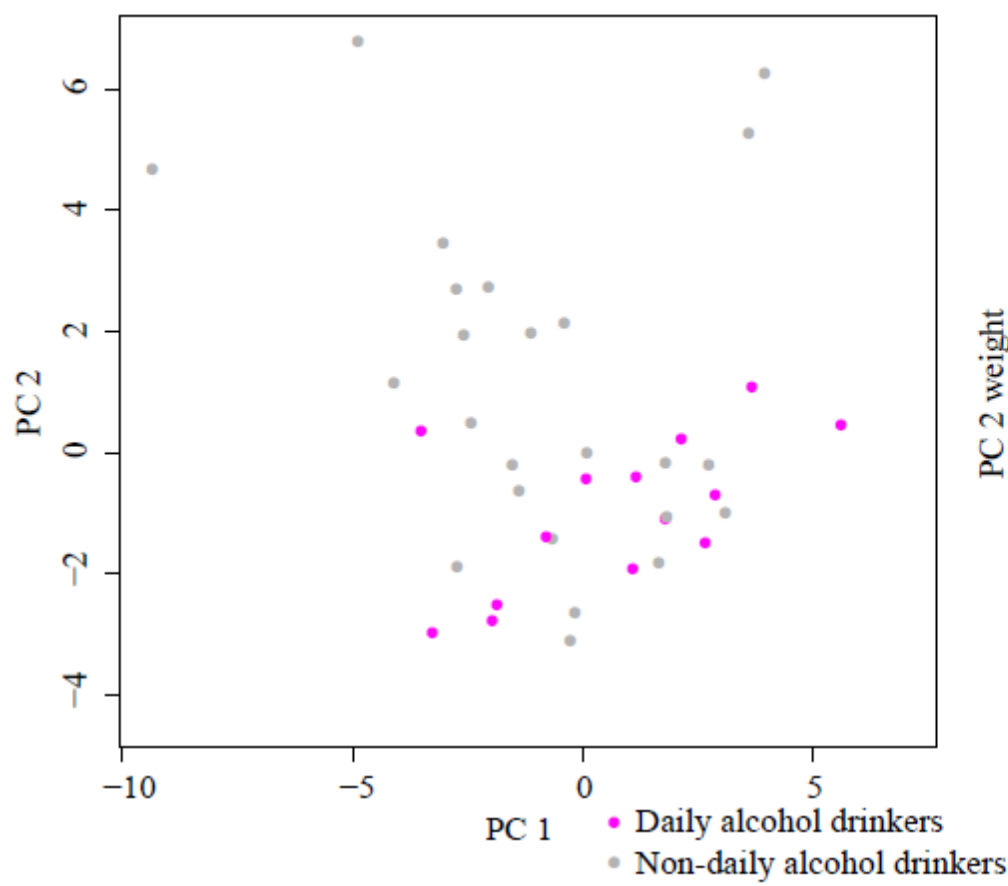

C

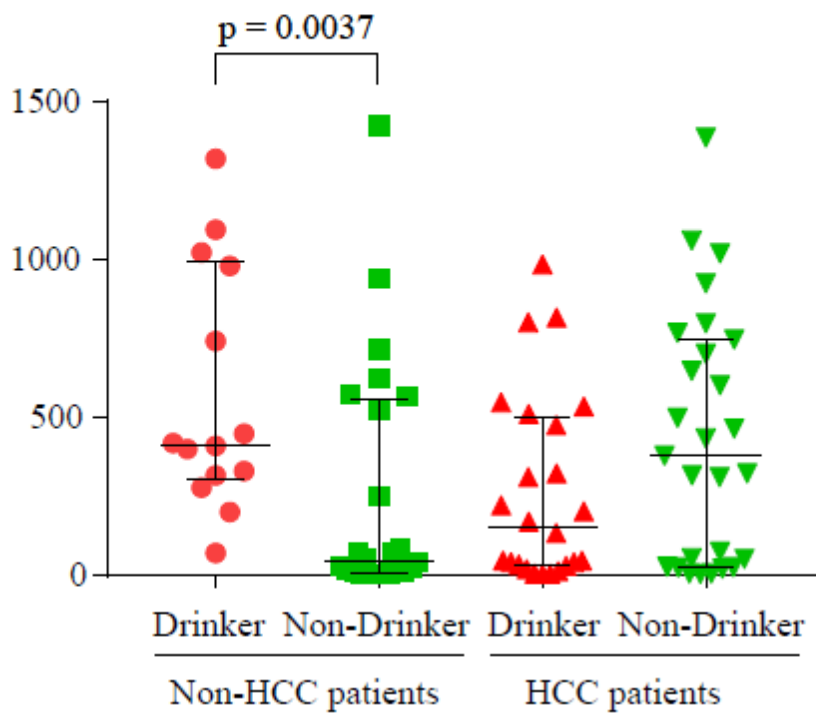

B

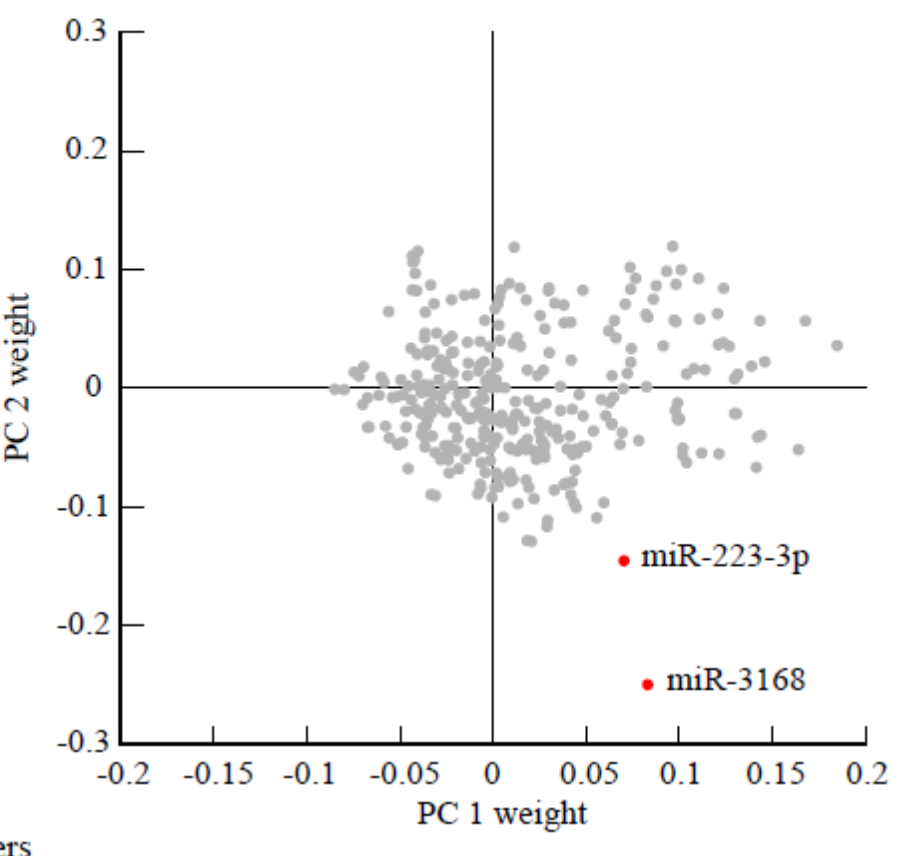

D

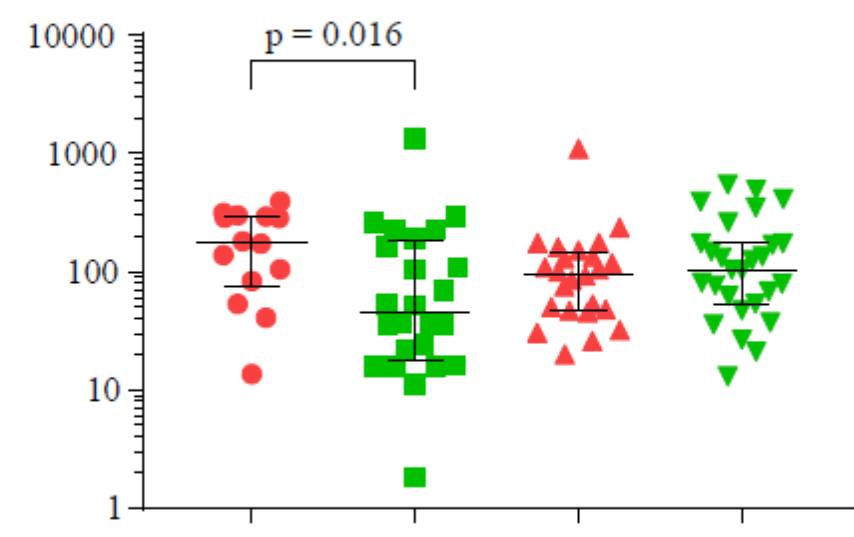

Drinker Non-Drinker Drinker Non-Drinker

Non- $\mathrm{HCC}$ patients $\mathrm{HCC}$ patients

\section{Figure 2}

Daily alcohol consumption altered the blood exosomal miRNA profiles of HBV positive non-HCC patients (A) Distribution of daily alcohol drinkers and non-daily alcohol drinkers on PC1 and PC2. (B) The distribution of PC1 weight and the PC2 weight of each miRNA. Level comparisons of miR-3168 (C) and miR-223-3p (D) from daily alcohol drinkers and non-daily alcohol drinkers between HCC patients and nonHCC patients are demonstrated. 
A



D

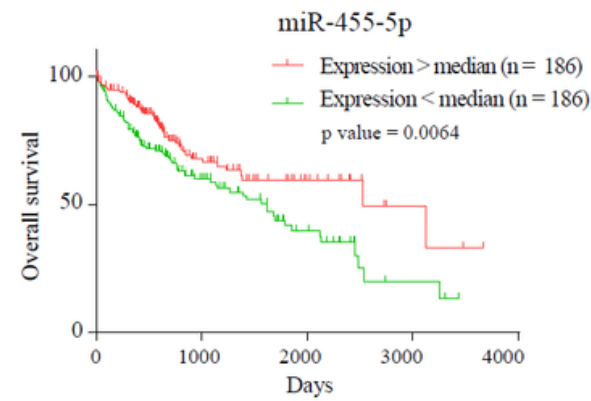

B

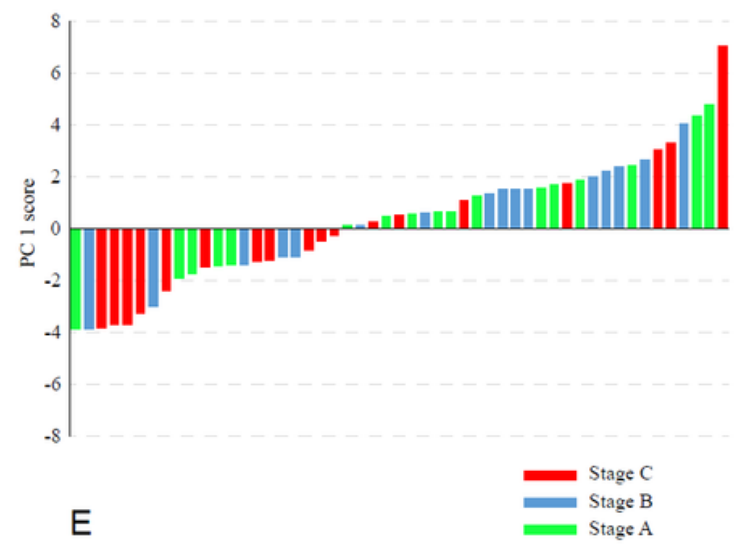

miR-30c-5p

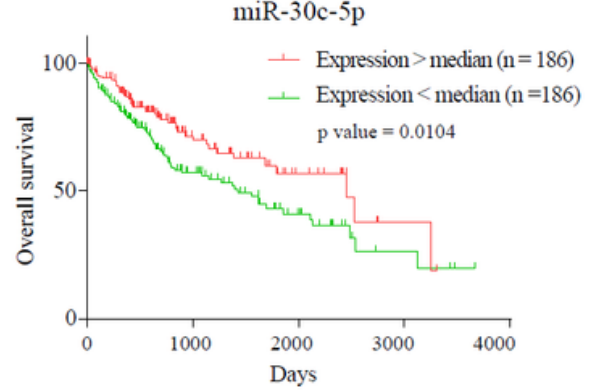

C

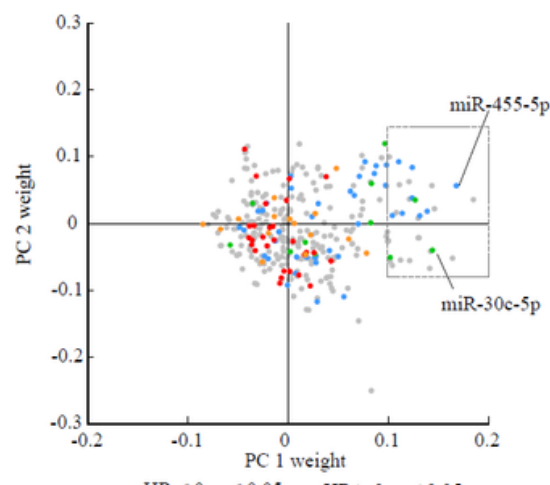



\section{Figure 3}

Blood exosomal miRNA profiles revealed the staging of tumor tissues (A) The distribution of HCC patients with stage $A$, stage $B$, and stage $C$ on PC1 and PC2. The green represents the patients with stage $A$, the blue represents the patients with stage $B$, and the red represents the patients with stage $C$. (B) Histogram displays PC1 scores of patients with stage A, stage $B$, and stage $C$. The histogram color is labeled in A. (C) The distribution of PC1 weight and the PC2 weight of each miRNA. The color labels the hazardous levels a Cox regression model based on TCGA data. (D) Kaplan-Meier statistical analyses on overall survival of two groups (high expression and low expression of miR-455-5p) were performed (E) Kaplan-Meier statistical analyses on overall survival of the two groups (high expression and low expression of miR-30c-5p) were performed.

A

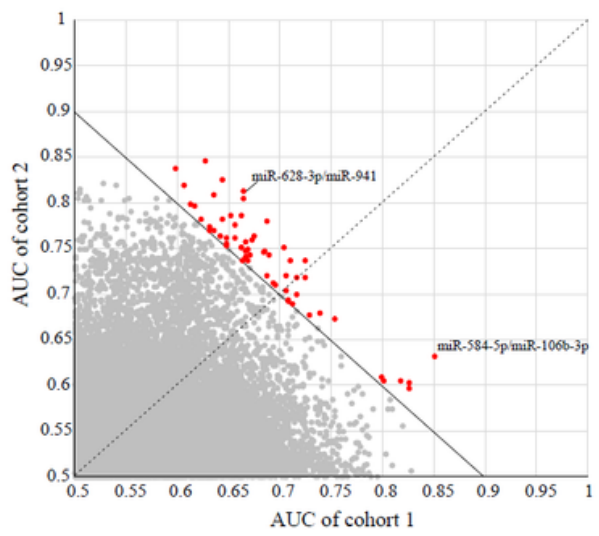

B

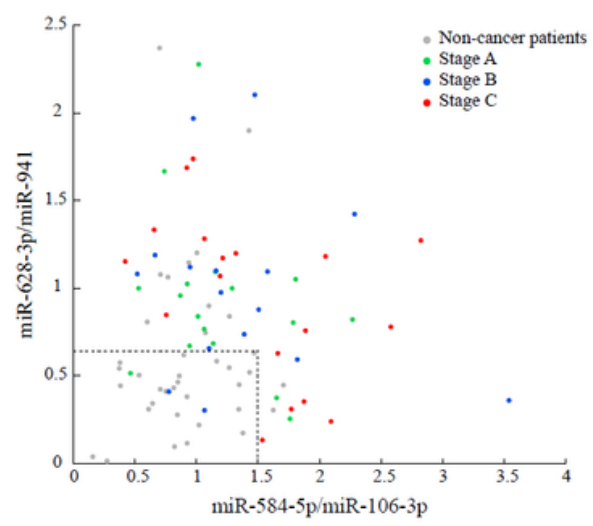

C



Figure 4 
The accuracy of biomarkers to classify HCC and non-HCC patients from blood exosomal miRNAs was affected by tumor staging and alcohol consumption habits (A) The AUCs of paired exosomal miRNAs in cohort 1 and cohort 2 are displayed. The distribution of the two paired blood exosomal miRNAs (miR-628$3 p / m i R-941$ and miR-584-5p/miR-106-3p) was colored according to tumor stage (B) and alcohol consumption habits (C).

\section{Supplementary Files}

This is a list of supplementary files associated with this preprint. Click to download.

- SupplementaryFiles.pdf 